\title{
Índice modificado de esfuerzo en cuidadores informales de personas con Enfermedad de Huntington en los Municipios de Algarrobo, Ariguaní y San Angel. Magdalena, Colombia
}

\section{Sandra Patricia Misnaza Castrillón ${ }^{1}$, Armenta-Restrepo Analidis ${ }^{2}$}

\author{
Magister en administración en salud. Profesional especializado Subdirección de Prevención, Vigilancia y Control en Salud Pública. Grupo de Enfermedades \\ Crónicas no Transmisibles. Instituto Nacional de Salud. \\ 2 Especialista en psicología clínica en niños y adolescentes. Contratista Subdirección de Análisis del Riesgo en Salud Pública. Instituto Nacional de Salud.
}

Fecha de recepción agosto 182016 Fecha de correcciones octubre 19 de 2016 Fecha de aceptación septiembre 30 de 2016

Fecha de publicación enero 31 de 2017

\begin{abstract}
Resumen
La Enfermedad de Huntington (EH) es una enfermedad genética, crónica con inicio de síntomas en la edad media de la vida. El cuidador es la persona más vulnerable, su bienestar dependerá de la gravedad de los síntomas del enfermo, los apoyos sociales y la percepción sobre el balance entre la carga y los beneficios. Métodos: estudio descriptivo mediante aplicación del índice modificado de esfuerzo del cuidador de 23 cuidadores en los municipios de Algarrobo, Ariguaní y San Angel. Resultados: El 82,6\% de los cuidadores eran mujeres, la media de edad fue 32,1 años. El índice modificado de esfuerzo de los cuidadores presentó una media de 9,37 y una mediana de 13; en promedio, el índice de mayor puntuación fue el esfuerzo físico. La distribución del índice de acuerdo al riesgo fue alto para el $39,1 \%$, medio para el $34,8 \%$ y bajo para el 26,1\%. Discusión: la mayor proporción de cuidadores presentó riesgo considerado alto, dado por la carga del cuidado en la EH.

Palabras clave: cuidador familiar, sobrecarga cuidador, enfermedad de Huntington, cuidados informales.

Copyright $\odot$ Facultad de Ciencias de la Salud de la Universidad Tecnológica de Pereira. 1995-2017. Todos los derechos reservados ${ }^{*}$
\end{abstract}

Modified index of effort of informal caregivers of people with Huntington's Disease located in the municipalities of Algarrobo, Ariguaní and San Angel. Magdalena, Colombia

\section{Abstract}

Huntington's disease (HD) is a chronic and genetic disease that starts in middle age. The caregiver is the most vulnerable person, his wellbeing will depend on the severity of the patient's symptoms, social support and the perception between balance of the burden and the benefits. Methods: A descriptive study was done applying an instrument with social, demographic and modified caregiver strain index variables to 23 caregivers in the municipalities of Algarrobo, Ariguaní and San Angel. Results: 82.6\% of caregivers were women, whose mean age was 32.1 years old. The modified index effort of caregivers had an average of 9.37 and a median of 13; in average the highest score index was physical effort. The index distribution according to risk was higher for $39.1 \%$, medium for $34.8 \%$, and low for $26.1 \%$. Discussion: The highest proportion of caregivers had a high risk; this may be because the care burden of HD patients and other chronic diseases involves a multidimensional construct that include epidemiological, economic and social perspective.

Key words: family caregiver, caregiver strain, Huntington disease, informal care

Copyright $\odot$ Facultad de Ciencias de la Salud de la Universidad Tecnológica de Pereira. 1995-2017. All rights reserved ${ }^{\circ}$.

\section{Introducción}

El síndrome de carga emocional se caracteriza principalmente por sentimientos de estrés y tristeza, además de agotamiento mental y ansiedad frente al cuidado. También está relacionado con dificultades en la salud física, debido a una acumulación de estresores frente a los que el cuidador se encuentra desprovisto de estrategias adecuadas de afrontamiento para adaptarse a la situación, por ejemplo, actividades diarias que exigen una dependencia parcial o total del paciente hacia su cuidador (aseo personal, vestido, alimentación, entre otras), cambios conductuales del paciente, altos costos económicos y limitación de actividades propias que generen satisfacción personal [1]; dicha dependencia además de incrementar los niveles de estrés, provoca alteraciones no sólo en el enfermo sino también en la familia, ya que su aparición demanda una redistribución de los roles familiares [2].

Por ello, la familia es una de las fuentes importantes de apoyo y cuidado del paciente, sin embargo dentro de ella, no todos los integrantes asumen de igual manera el cuidado de sus enfermos, por lo que los miembros que ocupan la máxima responsabilidad en el cuidado se denominan "cuidadores familiares"; del mismo modo, las personas que cumplen esta función, como el personal de enfermería, son llamados "cuidadores profesionales" $[3,4]$.

El cuidador se convierte en eje central de la familia y es la persona más vulnerable, su bienestar depende de la gravedad de los síntomas del enfermo, de los apoyos sociales de que disponga y de la percepción que tiene sobre el balance entre la carga que le supone y los beneficios que obtiene [5].

Entre las consecuencias psicológicas que sufren los cuidadores se incluyen desorientaciones, sentimientos de inadecuación, apatía, perdida de la autoestima y motivación. Dentro de la alteración cognitiva bajo los efectos del estrés, el pensamiento es confuso, la memoria olvidadiza y la concentración sufre deterioro, debido a que se limita el foco de atención del individuo afectando la calidad de decisiones. Entre los efectos conductuales se presenta excesiva ingestión de alimentos, pérdida de apetito, consumo excesivo de bebidas alcohólicas, conductas compulsivas y tendecia a sufrir accidentes [6].

La Enfermedad de Huntington (EH) o Corea de Huntington es una enfermedad crónica, neurodegenerativa que se transmite de generación en generación con inicio en la edad media de la vida[7] y se caracteriza por los siguientes síntomas: en el área motora se observan movimientos coreiformes involuntarios, atetosis, discinesia, ataxia, disartria y movimientos sacádicos anormales de los ojos [8,9]; en el área cognitiva, se afectan la memoria, atención y concentración, flexibilidad cognitiva, abstracción, planificación y generación de secuencias, fluencia verbal y demencia $[10,11]$; en cuanto a síntomas psiquiátricos y conductuales se encuentran la depresión, apatía, euforia, psicosis, irritabilidad, impulsividad, agresión 
y labilidad emocional [12,13]; y en el área funcional, alteraciones en el sueño y alimentación y mal funcionamiento en actividades de la vida diaria [14].

La prevalencia de la $\mathrm{EH}$ a nivel mundial se encuentra en 2,71 por 100.000 habitantes [15]. En Colombia es una enfermedad huérfana ya que se presenta en menos 1 por cada 5.000 personas [16].

El cuidado de un individuo afectado por EH puede resultar muy problemático debido a las complicaciones que surgen, a la variedad de síntomas de la enfermedad y al lento desarrollo; adicionalmente, se suman tareas que previamente eran llevadas a cabo por el paciente, tales como ganar dinero, cuidar de los hijos, labores domésticas, etc [13].

Teniendo en cuentalas características crónicas y neurodegenerativas de la $\mathrm{EH}$ y el papel de los familiares como cuidadores de muchos de estos pacientes, este estudio tuvo como objetivo estimar la sobrecarga experimentada por los cuidadores de personas con $\mathrm{EH}$ con el fin de generar información que permita realizar intervenciones que aporten a la mejora de calidad de vida de las personas con la enfermedad y sus familias.

\section{Métodos}

Estudio descriptivo transversal en una población constituida por 23 personas cuidadoras de pacientes con enfermedad de Huntington en los municipios de Algarrobo, Ariguaní y San Angel en el departamento del Magdalena, quienes fueron entrevistadas en el mes de agosto de 2015. No se realizó cálculo de muestra ya que todas las personas cuidadoras identificadas fueron entrevistadas.

La recolección de la información se realizó por medio de visitas domiciliarias a familias previamente identificadas como familias con personas afectadas por la enfermedad de Huntington en los registros de caracterización y localización de la discapacidad de las direcciones locales de salud de los municipios de Ariguaní y San Angel. En las visitas se aplicó el instrumento MCSI por sus siglas en inglés (modified caregiver strain index), el cual permite determinar el estrés, la idoneidad, la salud física y emocional de los cuidadores de enfermos crónicos [17], consta de 13 preguntas que evalúan varios grupos de temas entre ellas: el empleo, situación financiera, componente físico, componente social y tiempo [18].

Se realizó prueba piloto del instrumento en campo una semana antes del desplazamiento, ajustando el constructo de algunas preguntas de variables sociales y demográficas y definiendo el instrumento MCSI sobre el Test de Zarit ya que dicho test presentó dificultades en los encuestados porque la calificación (1 a 5) ofrecía muchas opciones de respuesta.

Las variables de estudio fueron cualitativas nominales e incluyeron variables sociales, demográficas y las incluidas en el MCSI.

El plan de análisis se realizó mediante estadística descriptiva en términos de persona y lugar y utilizando el programa Excel $^{\circledR}$. Para la interpretación de cada pregunta del índice MCSI se dio una calificación entre cero y dos $(0=$ No, $1=$ A veces, $2=$ Siempre $)$ de acuerdo a los recomendado por los autores [18].

Los resultados de los índices de esfuerzo fueron categorizados por percentiles para estimar el riesgo y fueron interpretados de acuerdo al estrés o esfuerzo que representaran (ver cuadro 1).

Consideraciones éticas

Esta investigación de acuerdo con la resolución 008430 de 1993 del Ministerio de Salud y la ley 84 de 1989, es de riesgo mínimo. Se solicitó el consentimiento informado en todos los casos.

Este estudio contempló dentro del diseño los principios metodológicos para salvaguardar el interés de la ciencia y el respeto a los derechos de las personas. En los procedimientos realizados no se intervino a alguna persona, no se violó el derecho a la privacidad y los resultados se presentan de manera agrupada.

\section{Resultados}

Tabla 1. Interpretación de los percentiles del índice modificado de esfuerzo del cuidador

\begin{tabular}{ccc}
\hline Percentil & Valor & Interpretación \\
\hline $\mathbf{2 5}$ & $<8,5$ & Riesgo bajo \\
$\mathbf{5 0}$ & 8,6 a 13 & Riego medio \\
$\mathbf{7 5}$ & 13,1 a 16,5 & Riesgo alto
\end{tabular}

Fueron entrevistados 23 cuidadores en 10 familias en las cuales al menos un miembro presentaba Enfermedad de Huntington, el 82,6\% $(n=19)$ de los cuidadores fueron del sexo femenino, la media de edad fue 32,1 años, la mediana 36, el límite inferior 17 y el superior 77 (Tabla 1).

En cuanto al municipio de residencia de los entrevistados, ocho residían en Ariguaní, 13 en San Angel y dos en Algarrobo, municipio vecino de San Angel y que fue visitado por rumores de un caso de una persona de sexo femenino con EH.

El nivel de escolaridad fue en su mayoría primaria (52,2\%), seguido de secundaria y educación superior con $17,4 \%$ cada uno, el $13 \%$ no refirieron ningún grado de escolaridad.

De las 23 personas entrevistadas, siete $(30,4 \%)$ no tenían conocimiento acerca de la enfermedad, sus síntomas y modo de transmisión; una persona refirió haber recibido entrenamiento para manejar la enfermedad, específicamente en lo relacionado a nutrición y una persona refirió recibir remuneración por el cuidado del enfermo.

En cuanto al grado de parentesco, cinco personas $(21,7 \%)$ no presentaban parentesco con las personas con $\mathrm{EH}$, dos (8,7\%) eran parientes en segundo grado y $16(69,6 \%)$ fueron parientes en primer grado.

Tabla 2. Índice promedio modificado de esfuerzo del cuidador por pregunta. Magdalena, 2015

\begin{tabular}{clc}
\hline $\begin{array}{c}\text { Número de } \\
\text { la pregunta }\end{array}$ & \multicolumn{1}{c}{ Tema central de la pregunta } & Promedio \\
\hline $\mathbf{1}$ & Sueño perturbado & 0,95 \\
$\mathbf{2}$ & Cuidado presenta inconvenientes & 1,05 \\
$\mathbf{3}$ & Cuidado implica esfuerzo físico & 1,45 \\
$\mathbf{4}$ & Cuidado limita & 1,00 \\
$\mathbf{5}$ & Ajustes familiares & 0,64 \\
$\mathbf{6}$ & Cambios en planes personales & 1,09 \\
$\mathbf{7}$ & Otras demandas de tiempo & 0,77 \\
$\mathbf{8}$ & Ajustes emocionales & 0,73 \\
$\mathbf{9}$ & Comportamientos molestos & 0,59 \\
$\mathbf{1 0}$ & Deterioro de la persona con EH & 1,36 \\
$\mathbf{1 1}$ & Ajustes en trabajo & 0,95 \\
$\mathbf{1 2}$ & Esfuerzo financiero & 1,18 \\
$\mathbf{1 3}$ & Estoy completamente abrumado & 1,23 \\
\hline
\end{tabular}

En cuanto al índice modificado de esfuerzo de los cuidadores y las preguntas que lo componen, en promedio, la respuesta que recibió mayor puntuación fue acerca del esfuerzo físico (ver tabla 2).

Tabla 3. Estadísticos descriptivos del índice modificado de esfuerzo del cuidador. Magdalena, 2015

\begin{tabular}{cccccccc}
\hline \multicolumn{7}{c}{ Sobrecarga a cuidadores informales } \\
Media & DS & Mediana & Moda & Rango & Min & Max & CV \\
\hline $\mathbf{9 , 3 7}$ & 5,63 & 13 & 13 & 19 & 3 & 22 & $43 \%$ \\
\hline
\end{tabular}

El índice modificado de esfuerzo de los cuidadores presentó una media de 9,37 y una mediana de 13 (ver tabla 3). 
Tabla 4. Índice modificado de esfuerzo del cuidador y características sociodemográficas de cuidadores primarios de personas con Enfermedad de Huntington. Magdalena, 2015

\begin{tabular}{llccl}
\hline Municipio & Sexo & Edad & MCSI & Interpretación \\
\hline San Angel & Femenino & 22 & 3 & Riesgo bajo \\
Ariguaní & Femenino & 24 & 4 & Riesgo bajo \\
Ariguaní & Masculino & 17 & 5 & Riesgo bajo \\
San Angel & Femenino & 49 & 5 & Riesgo bajo \\
San Angel & Femenino & 40 & 7 & Riesgo bajo \\
Ariguaní & Masculino & 24 & 8 & Riesgo bajo \\
Ariguaní & Femenino & 18 & 9 & Riesgo medio \\
San Angel & Femenino & 26 & 10 & Riesgo medio \\
Ariguaní & Femenino & 54 & 11 & Riesgo medio \\
Ariguaní & Femenino & 36 & 12 & Riesgo medio \\
Ariguaní & Masculino & 39 & 13 & Riesgo medio \\
San Angel & Femenino & 32 & 13 & Riesgo medio \\
San Angel & Femenino & 70 & 13 & Riesgo medio \\
San Angel & Masculino & 63 & 13 & Riesgo medio \\
San Angel & Femenino & 52 & 14 & Riesgo alto \\
San Angel & Femenino & 23 & 15 & Riesgo alto \\
San Angel & Femenino & 18 & 16 & Riesgo alto \\
San Angel & Femenino & 29 & 17 & Riesgo alto \\
San Angel & Femenino & 37 & 18 & Riesgo alto \\
Ariguaní & Femenino & 77 & 19 & Riesgo alto \\
Algarrobo & Femenino & 26 & 20 & Riesgo alto \\
Algarrobo & Femenino & 37 & 22 & Riesgo alto \\
San Angel & Femenino & 28 & 22 & Riesgo alto \\
\hline El $39,1 \%$ de & entevista & & \\
\hline
\end{tabular}

El 39,1\% de los entrevistados presentó riesgo por encima del percentil 75 e interpretado como alto, 34,8\% presentó riesgo medio y $26,1 \%$ riesgo bajo. La distribución de los índices modificados de esfuerzo de acuerdo al riesgo y a características sociodemográficas se observa en la tabla 4.

Tabla 5. Proporción de riesgo de acuerdo al índice modificado de esfuerzo del cuidador por municipio de residencia. Magdalena, 2015

\begin{tabular}{lccccccc}
\hline Municipio & $\begin{array}{c}\text { Riesgo } \\
\text { alto }\end{array}$ & $\mathbf{\%}$ & $\begin{array}{c}\text { Riesgo } \\
\text { bajo }\end{array}$ & $\begin{array}{c}\text { Riesgo } \\
\text { medio }\end{array}$ & $\begin{array}{c}\text { Total } \\
\text { general }\end{array}$ \\
\hline Algarrobo & 2 & 100 & 0 & 0 & 0 & 0 & 2 \\
Ariguaní & 1 & 12,5 & 3 & 37,5 & 4 & 50 & 8 \\
San Angel & 6 & 46,2 & 3 & 23,1 & 4 & 30,8 & 13 \\
Total & $\mathbf{9}$ & $\mathbf{3 9 , 1}$ & $\mathbf{6}$ & $\mathbf{2 6 , 1}$ & $\mathbf{8}$ & $\mathbf{3 4 , 8}$ & $\mathbf{2 3}$ \\
\hline
\end{tabular}

En los cuidadores residentes en el municipio de San Angel se presentó la mayor proporción de riesgo alto (ver tabla 5).

\section{Discusión}

La mayor proporción de cuidadores fue de sexo femenino y puede estar explicada por los patrones culturales de Colombia y en especial de la región Atlántica, también por características relacionadas con el trabajo, que predisponen principalmente al género femenino a cumplir el rol de cuidador [19], estos resultados concuerdan con lo expuesto en otros estudios de cuidadores [20-25].

La media de edad de los cuidadores fue de 32 años, edad laboralmente activa en la cual muchos de los cuidadores ya podrían tener hijos y familia que implicarían demandas adicionales de tiempo. La edad reportada fue menor a la otros estudios sobre cuidadores de personas con enfermedades crónicas [20-21,25].

El nivel de escolaridad fue en su mayoría primaria, resultados que concuerdan por lo descrito por Yonte en 2010 (20) pero no con Jofre en el mismo año, donde la mayor proporción de cuidadores presentó nivel de educación media [22]. De las personas entrevistadas, una persona refirió recibir remuneración por el cuidado del enfermo, lo cual podría explicarse por el grado de parentesco de la mayoría de los cuidadores (primer grado). Se ha documentado que la mayoría de los cuidadores informales no reciben remuneración por estas tereas [22].

El grado de consanguinidad de los cuidadores concuerda con otros estudios, siendo el primer grado de consanguinidad el que se presenta con mayor frecuencia $[20,24]$.

La pregunta que implicaba el esfuerzo físico como tema central de la sobrecarga del cuidador fue la que en promedio presentó mayor puntuación, lo cual puede estar explicado con las características neurodegenerativas de la $\mathrm{EH}$, debido a las complicaciones que surgen, a la variedad de síntomas de la enfermedad, y al lento desarrollo [26], lo cual implicaría entre otros, que en la medida en que la enfermedad avanza, el esfuerzo físico será mayor.

Otra de las preguntas con alta puntuación promedio fue la relacionada con el deterioro de la persona con $\mathrm{EH}$ y los sentimientos de impotencia en el cuidador al no observar mejoría, lo cual se explicaría porque el cuidador responde emocionalmente de una manera compleja con afectos positivos y negativos, pueden experimentar sentimientos de pena, impotencia, agresividad, culpabilidad, ambivalencia (deseos de ayudar y al mismo tiempo, sentir al enfermo como una carga o desear que se muera para que no sufra), injusticia y temor al futuro; como respuesta pueden sufrir un derrumbe psicológico (depresión, ansiedad) o físico (enfermar) [26].

Al respecto, un estudio realizado en Estados Unidos y Canadá en 2009, describió entre las preocupaciones de los cuidadores de EH y otros cuidadores de enfermedades crónicas la sensación de aislamiento social, sentimientos de ira en relación con los cambios en las funciones debido al deterioro cognitivo y labilidad emocional del enfermo, sentimientos de ansiedad en relación a la adopción de roles poco habituales dentro de la familia, preocupación por no cumplir los propios sueños, el riesgo latente en descendientes por desarrollar la enfermedad, entre otros [27-29].

La pregunta relacionada con el efecto (sentirse abrumado) de brindar cuidado también presentó una alta puntuación promedio, posiblemente debido a la acumulación de estresores ya que el cuidador se encuentra desprovisto de estrategias adecuadas de afrontamiento para adaptarse a la situación (1).

Un estudio realizado en Chile en 2010 con la metodología de escala de sobrecarga del cuidador de Zarit, presentó sobrecarga leve en cuidadores de pacientes dependientes ingresados en el programa de atención domiciliaria del Servicio de Salud [25], sin embargo los datos no son comparables con los resultados de este estudio ya que la metodología y enfermedad de base de los pacientes objeto del cuidado son diferentes.

Con respecto a los cuidadores informales, aunque específicamente en adultos mayores, Zarit menciona que son las familias las que con mayor frecuencia se encargan del cuidado de los ancianos dependientes. El autor considera que la responsabilidad no puede recaer sólo en la familia; por la intensidad y costo de las cargas, por lo que sugiere una responsabilidad compartida, creativa, donde se consideren las necesidades de la persona cuidada y del cuidador, por lo que es necesario realizar estudios que conduzcan al logro de mejores estrategias de intervención $[21,30]$.

Con base en lo anterior, es importante recalcar que cuando la EH progresa, la familia necesita introducir cuidadores externos, preferiblemente cercanos al enfermo, ya que los pacientes necesitan una estabilidad en su entorno y normalmente, no reaccionan bien a los cambios. Por ello, es importante que haya una continuidad en los cuidadores. El apoyo psicológico y el entrenamiento de los cuidadores externos es muy importante para mantener el equilibrio psicológico de las personas con enfermedad de Huntington [26]. 
Dado que la carga del cuidado en la $\mathrm{EH}$, así como las demás enfermedades crónicas implica un constructo multidimensional que incluye la perspectiva epidemiológica (relación con la frecuencia y valoración de consecuencias en años de vida y pérdidas funcionales), la económica (impacto sobre consumo, ahorro, oferta de mano de obra, productividad y acumulación de capital humano) y la social (factores de desgaste, estrés, impacto familiar, limitación social y aspectos positivos de la experiencia), con múltiples interrelaciones entre sí, se requiere una mirada integral para examinar la carga del cuidado en la enfermedad crónica que incluya a los actores sociales afectados, pacientes, cuidadores familiares, familias, profesionales e instituciones de salud [31]. El alivio de la carga del cuidado de la enfermedad, a partir de medir el impacto y la efectividad de las intervenciones que se propongan, debe hacerse con base en indicadores que hayan sido mundialmente valorados pero que se ajusten al referente nacional y local, y que permitan construir y validar modelos de atención integral para responder a los retos del país [31].

Los resultados de este estudio son un insumo importante para el diseño de intervenciones para los cuidadores ya que el cuidado puede tener efectos negativos y perjudiciales sobre estos, lo anterior de acuerdo a lo documentado en 2003 por Thorton y Travis [17], quienes además determinaron que el instrumento utilizado en este estudio, el MCSI es útil en la investigación de cuidado familiar [17]. Este índice proporciona mayor información no solo a los médicos tratantes, sino también al sistema de salud como tal sobre las capacidades de los cuidadores [32] de personas con enfermedades crónicas neurodegenerativas.

Entre las limitaciones se encuentra que el MCSI es un instrumento diseñado para evaluar la sobrecarga o esfuerzo en cuidadores principalmente de pacientes con enfermedades crónicas, sin embargo no existen aún estudios enfocados específicamente en la Enfermedad de Huntington. El MCSI no desglosa la puntuación para clasificar los resultados en términos de baja, moderada o alta sobrecarga del cuidador, solo indica " 0 " como ningún estrés o sobrecarga y " 26 " como sobrecarga extrema.

\section{Conflicto de interés}

Los autores manifiestan no tener ningún conflicto de interés

\section{Agradecimientos}

A las Alcaldías Municipales de San Angel y Ariguaní por el apoyo logístico, en especial a Carmen Cecilia Bermudez Orozco, referente de discapacidad del Municipio de San Angel y Leonardo Rafael Guzman Rivera, vigilante en salud del Municipio de Arguaní; también a la Secretaria Departamental de Salud del Magdalena, en especial a Carmen Rocio del Toro Ramos, Aixa Ayala Quiroz, Liceth Maria Parejo Gonzalez, Cary Luz Jimenez Guette y Nolvis Fonseca Muñiz, quienes acompañaron el trabajo en campo; a los profesionales especializados del Instituto Nacional de Salud Teodolinda Vega Montaño, Diana Marcela Ruiz Quiroz, Alfonso Campo Carey, Antonio José Bermudez y Flavio Garzon, que conformaron el equipo que recolectó la información clínica y genealógica, y en especial a las familias de Algarrobo, Ariguaní y San Angel que abrieron sus puertas para poder hacer posible esta investigación de campo.

\section{Referencias}

1. Freudenberguer H. Staff B. J Soc Issues. 1974;30:159-65

2. Parodi JF. EL Cuidador Del Paciente Adulto Mayor Dependiente. Perú: Copyright; 2006

3. Pecea y Galeon. "Palabras para el cuidador". [fecha de consulta: 22 de agosto de 2013]. Disponible en: http://www.pecea.galeon. com

4. Peinado A, Garcés E. Burnout en cuidadores principales de pacientes con Alzheimer: el síndrome del asistente desasistido. Anales de Psicología. 2004;14:83-93.
5. Roscoe LA, Corsentino E, Waatkins S, McCall M, SánchezRamos J. Well-being of family caregivers of persons with latestage Huntintong's disease: lessons in stress and coping. Health Commun. 2009; 24: 239-248

6. Macalopú C, Vargas G. Rol de ayuda de la enfermera y nivel de estrés del cuidador primario del adulto mayor dependiente. Hospital Belén de Trujillo.2014. [fecha de consulta: 14 de Enero de 2015] Disponible en: http://repositorio.upao.edu.pe/handle/ upaorep/921.

7. Roos R. Huntington's disease: a clinical review. Journal of Rare Disease. 2010;5:1-8

8. Jankovic J, Shannon KM. Movement disorders. En: Bradley WG, Daroff RB, Fenichel GM, Jankovic J, eds. Neurology in Clinical Practice.5th ed. Philadelphia: Butterworth-Heinemann (Elsevier) 2008; cap. 75: 2081-2122.

9. Devinsky O, D'Esposito M. Neurology of cognitive and behavioral disorders. New York: Oxford University Press, 2004.

10. Barquero MS, Gómez E. Trastornos cognitivos en pacientes con enfermedad de Huntington. Rev Neurol. 2001; 32: 1067-1071.

11. Martínez-Larrea, JA. Síndromes depresivos asociados a deterioro cognitivo. An Sist Sanit Navar 2002; 25 (Supl. 3):105-115.

12. Ribaï $\mathrm{P}, \mathrm{Nguyen} \mathrm{K}, \mathrm{Hahn}-\mathrm{Barma} \mathrm{V}$ et al. Psychiatric and cognitive difficulties as indicators of juvenile Huntington disease onset in 29 patients. Arch Neurol 2007; 64: 813-819.

13. Smolina E. Psychosocial impact of Huntington's disease on families and spouses from the perspective of the family systems theory. Studies by Undergraduate Researchers at Guelph 2007; 1: 51-62.

14. HDSA (Huntington's disease Society of America). Huntington's Disease, 2008. Disponible en http://www.hdsa.org/

15. Pringsheim K, Wiltshire K, Day L, Dykeman J, Steeves T, Jette $\mathrm{N}$. The incidence and prevalence of Huntingtong's disease: A systematic review and meta-analysis. Movement Disorders. 2012;27:1075-1027

16. República de Colombia. Ley 1438 de 2011. Por medio de la cual se reforma el Sistema General de Seguridad Social en Salud y se dictan otras disposiciones. Enero 19 de 2011

17. Thorton M, Travis S. Analysis of the reliability of the modified caregiver strain index. Journal of gerontology: Social Science. 2003, 58B N2: S127-S132

18. Terry, M. The modified caregiver Strain Index. The Hartford Institute for Geriatric Nursing. 2007;14:65

19. Zambrano R, Ceballos P. Síndrome de carga del cuidador. Revista Colombiana de Pisquiatria. 2007;36:26S-39S

20. Yonte F, Urien Z, Martín M, Montero MR. Sobrecarga del cuidador principal. Nure Investigación. 2010;48:1-7

21. Florez E, Rivas E, Seguel F. Nivel de sobrecarga en el desempeño del rol del cuidador familiar de adulto mayor con dependencia severa. Ciencia y Enfermería. 2012; 18:29-41

22. Jofre V, Sanhueza O. Sobrecarga de cuidadores informales. Ciencia y Enfermería. 2010; 16:111-120

23. Perez M, Llibre J. Características sociodemográficas y nivel de sobrecarga de cuidadores de ancianos con enfermedad de Alzheimer. Revista Cubana de Enfermería. 2010;26:104-116

24. Dejo Vásquez M. Sentido de coherencia, afrontamiento y sobrecarga en cuidadores familiares de ancianos con enfermedad crónica. Avances en psicología latinoamericana. 2007;25:64-71

25. Espinoza K, Jofre V. Sobrecarga, apoyo social y autocuidado en cuidadores informales. Ciencia y Enfermería. 2012;18:23-30

26. Fernandez M, Grau C, Trigo P. Impacto de la enfermedad de Huntington en la familia. An. Sist. Sanit. Navar. 2012;35:295-307

27. Croog SH, Burleson JA, Sudilovsky A, Baume RM. Spouse caregivers of Alzheimer patients: problem responses to caregiver burden. Aging \& Mental Health. 2006;10:87-100 
28. Thomas M, Greenop K. Caregiver experiences and perceptions of stroke. Health SA Gesondheid. 2008;13:29-34

29. Loue S, Lowder JL, Buzney SJ, Buzo AM. Caring for an adult child with cognitive disabilities: meeting the dual needs of an adult and child. Care Management Journals. 2006;7:191-198

30. Zarit SH, Davey A, Edwards AB, Femia EE, Jarrott SE. Family Caregiving: Research Findings and Clinical Implications. En: Bellack AS, Hersen M, editors y Edelstein BA, editor volumen. Comprehensive clinical psychology. Vol. 7, Clinical geropsychology. Oxford, UK: Elsevier Science; 1998. p. 499-523.

31. Sánchez B, Carrillo G, Barrera L, Chaparro L. Carga del cuidado de la enfermedad crónica no transmisible. Aquichan. 2013;13: 247-260.

32. Onega L. Helping those who help others. The modified caregiver strain index. AJN. 2008; 108:62-69 